\title{
NONLINEAR EIGENVALUE PROBLEM AND SINGULAR VARIATION OF DOMAINS
}

\author{
Dedicated to Professor Hirokı Tanabe on his 60 th birthday
}

By Tatsuzo Osawa and Shin Ozawa

\section{Introduction.}

Recently we have huge amount of research papers concerning semi-linear elliptic boundary value problems. See, for example Berestychi-Lions-Peletier [3], Dancer [4], Lin [5], Ni-Serrin [6], Rabinowitz [14], Wang [15] and the literatures cited there.

In this paper we want to discuss the following quantitative result for nonlinear eigenvalue problem with the Robin condition.

Let $\Omega \subset R^{2}$ be a bounded domain with smooth boundary $\partial \Omega$. Let $w$ be a fixed point in $\Omega$. Let $B(\varepsilon ; w)$ denote the ball of center $w$ with radius $\varepsilon$. We remove $B(\varepsilon ; w)$ from $\Omega$ and we get $\Omega_{\varepsilon}=\Omega \backslash \overline{B(\varepsilon ; w)}$. We write $B(\varepsilon ; w)$ as $B_{\varepsilon}$.

Fix $p \in(1, \infty)$. We fix $k>0$. We put

$$
\lambda(\varepsilon)=\inf _{u \in X}\left(\int_{\Omega_{\varepsilon}}|\nabla u|^{2} d x+k \int_{\partial B_{\varepsilon}} u^{2} d \sigma_{x}\right),
$$

where $X=\left\{u \in H^{1}\left(\Omega_{\varepsilon}\right), u=0\right.$ on $\partial \Omega$ and $u \geqq 0$ in $\left.\Omega_{\varepsilon},\|u\|_{L p+1\left(\Omega_{\varepsilon}\right)}=1\right\}$. We see that there exists at least one solution $v_{\varepsilon}$ of the above problem which attains (1.1)s.

We see that $v_{\varepsilon}$ satisfies

$$
\begin{array}{ll}
-\Delta v_{\varepsilon}(x)=\lambda(\varepsilon) v_{\varepsilon}(x)^{p} & x \in \Omega_{\varepsilon} \\
v_{\varepsilon}(x)=0 & x \in \partial \Omega \\
k v_{\varepsilon}(x)+\frac{\partial}{\partial \nu_{x}} v_{\varepsilon}(x)=0 & x \in \partial B(\varepsilon ; w) .
\end{array}
$$

Here $\partial / \partial \nu_{x}$ denotes the derivative along the exterior normal vector with respect to $\Omega_{\varepsilon}$.

We write

$$
\lambda=\inf _{u \in Y} \int_{\Omega}|\nabla u|^{2} d x,
$$

where $Y=\left\{u ; u \in H_{0}^{1}(\Omega), u \geqq 0\right.$ in $\left.\Omega,\|u\|_{L^{p+1}(\Omega)}=1\right\}$. There exists at least one

Receıved March 10, 1992. 
positive solution $v$ which attains (1.2). It satisfies $-\Delta v=\lambda v^{p}$ in $\Omega, v=0$ on $\partial \Omega$.

Main result of this paper is the following.

THEOREM. Assume that the positive function $\boldsymbol{u}_{\varepsilon}$ which attains $(1.1)_{\varepsilon}$ is unique for $0<\varepsilon \ll 1$. Assume also that the positive function $\boldsymbol{u}$ which attains (1.2) is unique. Assume that $\operatorname{Ker}\left(\Delta+p \lambda(\varepsilon) \boldsymbol{u}_{\varepsilon}^{p-1}\right)=\{0\}$ for $0<\varepsilon \ll 1$. Assume that $\left\|\boldsymbol{u}_{\varepsilon}^{p}-\boldsymbol{u}^{p}\right\|_{L} \tilde{q}_{\left(\Omega_{\varepsilon}\right)} \rightarrow 0$ for some $\tilde{q}>1$ and $\sup _{\varepsilon} \varepsilon^{2}\left\|\boldsymbol{u}_{\varepsilon}^{p}\right\|_{L q\left(\Omega_{\varepsilon}\right)}$ is finite for fixed large $q$. Under these assumptions, we have

$$
\lambda(\varepsilon)-\lambda=2 \pi k \varepsilon \boldsymbol{u}(w)^{2}+o(\varepsilon) .
$$

Remark. Here the operator $\Delta+p \lambda(\varepsilon) \boldsymbol{u}_{\varepsilon}^{p-1}$ means the operator associated with the boundary condition with respect to $(1.1)_{\varepsilon}$. The inequality $\lambda(\varepsilon) \leqq \lambda+O(\varepsilon)$ is easy to show. Let $\chi_{\varepsilon}(x)$ be the characteristic function of $\Omega_{\varepsilon}$. Then we put $F_{\varepsilon}(x)=\chi_{\varepsilon}(x) \boldsymbol{u}(x)$. Then,

$$
\begin{aligned}
\lambda(\varepsilon) & \leqq \\
& =\lambda+O\left(\int_{\Omega_{\varepsilon}}\left|\nabla F_{\varepsilon}\right|^{2} d x+\int_{\partial \Omega_{\varepsilon}} k F_{\varepsilon}(x)^{2} d \sigma_{x}\right) /\left(\int_{\Omega_{\varepsilon}} F_{\varepsilon}(x)^{p+1} d x\right)^{2 /(p+1)}
\end{aligned}
$$

There are many papers concerning eigenvalues of the Laplacian under singular variation of domains. See Ozawa [8], [9], [10], [11], Besson [2] and the literature cited there.

Our proof of Theorem is given by a systematic use of the Hadamard variational formula developed by Osawa [7] and the techniques in Ozawa [10]. The authors think that the techniques developed in this paper have wide class applications to investigation of semi-linear boundary value problems.

We quote the following theorem from Osawa [7]. It should be remarked that more general theorem is treated in [7].

Theorem ([7]). Fix $\varepsilon$. Assume that positive minimizer $u_{\varepsilon}$ associated with $(1.1)_{s}$ is unique and $\operatorname{Ker}\left(\Delta+p \lambda(\varepsilon) u_{\varepsilon}^{p-1}\right)=0$. Then,

$$
\frac{\partial}{\partial \varepsilon} \lambda(\varepsilon)=-\int_{\partial B_{\varepsilon}}\left(\left|\tilde{\nabla} u_{\varepsilon}\right|^{2}-(2 \lambda(\varepsilon) /(p+1)) u_{\varepsilon}^{p+1}-\left(k^{2}-k H_{1}\right) u_{\varepsilon}^{2}\right) d \sigma_{x},
$$

where $H_{1} \equiv-\varepsilon^{-1}$ is the first mean curvature of the boundary point with respect to the interior normal vector at $x$. Here $\tilde{\nabla}$ denotes the gradient operator on the tangent line.

Thus,

$$
\lambda(\varepsilon)-\lambda=\int_{0}^{s}\left(I_{1}(t)+I_{2}(t)+I_{3}(t)+I_{4}(t)\right) d t,
$$

where

$$
I_{1}(t)=-\int_{\partial B_{t}}\left|\tilde{\nabla} u_{t}\right|^{2} d \sigma_{x}, \quad I_{2}(t)=2 \int_{\partial B_{t}}(\lambda(t) /(p+1)) u_{t}^{p+1} d \sigma_{x},
$$




$$
I_{3}(t)=k^{2} \int_{\partial B_{t}} u_{t}^{2} d \sigma_{x}, \quad I_{4}(t)=+k \int_{\partial B_{t}} t^{-1} u_{t}^{2} d \sigma_{x} .
$$

\section{Preliminary Lemma.}

Lemma 2.1. Fix $L \in C^{\infty}\left(\partial B_{\varepsilon}\right)$. Let $u$ be the solution of

$$
\begin{array}{ll}
\Delta u(x)=0 & x \in \Omega \backslash \overline{B(\varepsilon ; w)} \\
u(x)=0 & x \in \partial \Omega \\
k u(x)+\frac{\partial}{\partial \nu_{x}} & u(x)=L(\theta) \quad x=w+\varepsilon(\cos \theta, \sin \theta) .
\end{array}
$$

Then, $u(x)$ satisfies

$$
\begin{aligned}
& |u(x)| \leqq C \varepsilon \operatorname{Max}_{\theta}|L(\theta)| \\
& |\operatorname{grad} u(x)| \leqq C\left(\|L\|_{L^{2}\left(S^{1}\right)}+\|L\|_{\left.C^{(3 / 4)+\sigma}\right)^{2(1+\alpha) / 3}\|L\|_{L^{\infty}\left(S^{1}\right)}^{(1-2 \alpha) / 3}+C\|L\|_{L^{\infty}\left(S^{1}\right)}}\right.
\end{aligned}
$$

for any $\alpha \in(0,1 / 2), \sigma>0$.

Proof. We put

$$
f(x)=a_{0} \log r+\sum_{\jmath=1}^{\infty}\left(b_{j} \cos j \theta+c_{\jmath} \sin \jmath \theta\right)(-j)^{-1} r^{-\jmath} .
$$

Then, it satisfies $\Delta f(x)=0 \quad x \in R^{2} \backslash \bar{B}_{\varepsilon}$. We expand $L(\theta)$ in a Fourier series

$$
L(\theta)=s_{0}+\sum_{j=1}^{\infty}\left(s_{j} \cos j \theta+t_{j} \sin j \theta\right) .
$$

Therefore,

$$
\begin{aligned}
& a_{0}=k^{-1} s_{0}\left(\log \varepsilon-k \varepsilon^{-1}\right)^{-1} \\
& b_{j}=k^{-1} s_{j}\left((-j)^{-1}-k^{-1} \varepsilon^{-1}\right)^{-1} \varepsilon^{j} \\
& c_{j}=k^{-1} t_{j}\left((-j)^{-1}-k^{-1} \varepsilon^{-1}\right)^{-1} \varepsilon^{\jmath} .
\end{aligned}
$$

We see that

$$
\left|f(x)_{\mid \curvearrowright \Omega}\right| \leqq C \varepsilon
$$

observing

$$
\sum_{j}\left(s_{j}^{2}+t_{\jmath}^{2}\right) \leqq C \max _{\theta} L(\theta)^{2}
$$

Then, we solve $\Delta v(x)=0, x \in \Omega$ and $v(x)=f(x)$ for $x \Subset \partial \Omega$. And we put

$$
L^{(2)}(\theta)=v(x)_{\mid x=w+\varepsilon(\cos \theta, \sin \theta)} .
$$

We solve

$$
\begin{aligned}
& \Delta f^{(2)}(x)=0 \quad x \in R^{2} \backslash \bar{B}_{\varepsilon} \\
& k f^{(2)}(x)+\frac{\partial}{\partial \nu_{x}} f^{(2)}(x)=L^{(2)}(\theta) .
\end{aligned}
$$


We continue this procedure, then we get $u(x)=f(x)-v(x)+f^{(2)}(x) \cdots$ satisfies (2.1). Observing this step, we get

$$
\begin{aligned}
|u(x)| & \leqq C\left(\left|s_{0}\right| \varepsilon+\sum_{j=1}^{\infty}\left(\left|s_{\jmath}\right|+\left|t_{\jmath}\right|\right)(-j)^{-1} \varepsilon^{\jmath+1} r^{-j}\right) \\
& \leqq C \varepsilon\left(\left|s_{0}\right|+\left(\sum_{j=1}^{\infty}\left(s_{j}^{2}+t_{j}^{2}\right)\right)^{1 / 2}\left(\sum_{j=1}^{\infty} j^{-2} \varepsilon^{2 \jmath} r^{-2 j}\right)^{1 / 2}\right)
\end{aligned}
$$

We use

$$
2 \pi s_{0}^{2}+\pi \sum_{j=1}^{\infty}\left(s_{j}^{2}+t_{j}^{2}\right)=\int_{0}^{2 \pi} L(\theta)^{2} d \theta \leqq 2 \pi \max _{\theta} L(\theta)^{2} .
$$

Therefore, we get the first part of (2.2). By the above construction of $u$ we see that

$$
\begin{aligned}
|\operatorname{grad} u(x)| & \left.\leqq C\left(\sum_{j=1}^{\infty}\left|s_{j}\right|+\left|t_{j}\right|\right)\left(r^{-(\jmath+1)} \varepsilon^{\jmath+1}\right)+C\left|s_{0}\right| \varepsilon r^{-1}\right) \\
& \leqq C\left(\left(\sum_{j=1}^{\infty}\left(j^{1+\alpha}\left(s_{j}^{2}+t_{\jmath}^{2}\right)\right)^{1 / 2}\left(\sum_{j=1}^{\infty} j^{-(1+\alpha)}(\varepsilon / r)^{2(\jmath+1)}\right)^{1 / 2}\right.\right.
\end{aligned}
$$

for $\alpha>0$.

We have the inequality

$$
\left(\sum_{j=1}^{\infty} j^{1+\alpha}\left(s_{j}^{2}+t_{j}^{2}\right)\right)^{1 / 2} \leqq\left(\sum_{j=1}^{\infty} j^{3 / 2}\left(s_{j}^{2}+t_{j}^{2}\right)\right)^{(1+\alpha) / 3}\left(\sum_{j=1}^{\infty}\left(s_{j}^{2}+t_{j}^{2}\right)\right)^{(1-2 \alpha) / 6}
$$

for $\alpha \in(0,1 / 2)$.

We know that $H^{3 / 4}\left(S^{1}\right)$-norm of $h$ is equivalent to the following norm. See Adams [1].

$$
\|h\|_{L^{2}\left(S^{1}\right)}+\left(\int_{S^{1}} \int_{S^{1}}|h(x)-h(y)|^{2}|x-y|^{-5 / 2} d x d y\right)^{1 / 2}
$$

Thus, we have

$$
\|h\|_{H^{3 / 4}\left(S^{1}\right)} \leqq C\left(\|h\|_{L^{2}\left(S^{1}\right)}+\|h\|_{C^{(3 / 4)+\sigma}\left(S^{1}\right)}\right)
$$

for any $\sigma>0$. Summing up these facts we get the second part of (2.2).

\section{Approximation of the Geen function.}

This section is heavily depend on the previous paper of one of the authors $[10]$. We introduce the following kernel $p_{\varepsilon}(x, y)$.

$$
p_{\varepsilon}(x, y)=G(x, y)+g(\varepsilon) G(x, w) G(w, y)+h(\varepsilon)\left\langle\nabla_{w} G(x, w), \nabla_{w} G(w, y)\right\rangle,
$$

where

$$
\left\langle\nabla_{w} a, \nabla_{w} b\right\rangle=\left.\sum_{\imath=1}^{2} \frac{\partial}{\partial \tilde{w}_{\imath}} a(\tilde{w}) \frac{\partial}{\partial \tilde{w}_{\imath}} b(\tilde{w})\right|_{\tilde{w}=w}
$$


for orthnomal frame $\left(w_{1}, w_{2}\right)$ of $\boldsymbol{R}^{2}$ and where

$$
g(\varepsilon)=-\left(\gamma-(2 \pi)^{-1} \log \varepsilon+(k 2 \pi)^{-1} \varepsilon^{-1}\right)^{-1}
$$

and

$$
h(\varepsilon)\left((2 \pi \varepsilon)^{-1}+(2 \pi)^{-1} k^{-1} \varepsilon^{-2}\right)=k^{-1} .
$$

Here

$$
\gamma=\lim _{x \rightarrow w}\left(G(x, w)+(2 \pi)^{-1} \log |x-w|\right) .
$$

Let $G_{\varepsilon}(x, y)$ be the Green function of the Laplacian in $\Omega_{\varepsilon}$ associated with the boundary conditions

$$
\begin{aligned}
& G_{\varepsilon}(x, y)=0 \quad x \in \partial \Omega \\
& k G_{\varepsilon}(x, y)+\frac{\partial}{\partial \nu_{x}} G_{\varepsilon}(x, y)=0 \quad x \in \partial B_{\varepsilon} .
\end{aligned}
$$

We put

$$
\begin{aligned}
& \boldsymbol{G}_{\varepsilon} f(x)=\int_{\Omega_{\varepsilon}} G_{\varepsilon}(x, y) f(y) d y \\
& \boldsymbol{P}_{\varepsilon} f(x)=\int_{\Omega_{\varepsilon}} p_{\varepsilon}(x, y) f(y) d y .
\end{aligned}
$$

We want to prove the following. We put $\boldsymbol{Q}_{\varepsilon} f(x)=\boldsymbol{P}_{\varepsilon} f(x)-\boldsymbol{G}_{\varepsilon} f(x)$.

There exists a constant $C$ independent of $\varepsilon$ such that

$$
\begin{aligned}
& \max _{x \in \partial B_{\varepsilon}}\left|k \boldsymbol{Q}_{\varepsilon} f(x)+\frac{\partial}{\partial \nu_{x}} \boldsymbol{Q}_{\varepsilon} f(x)\right| \leqq C \boldsymbol{\varepsilon}\|f\|_{L q\left(\Omega_{\varepsilon}\right)} \\
& \max _{x \in \partial B_{\varepsilon}}\left|\nabla \boldsymbol{Q}_{\varepsilon} f(x)\right| \leqq C \boldsymbol{\varepsilon}^{(1-2 \alpha) / 3}\|f\|_{L q\left(\Omega_{\varepsilon}\right)}
\end{aligned}
$$

for any $\alpha \in(0,1 / 2), q>8$.

Proof of (3.4), (3.5). Since $\boldsymbol{G}_{\varepsilon} f(x)$ satisfies the third boundary condition, then we have only to calculate

$$
k \boldsymbol{P}_{\varepsilon} f(x)+\frac{\partial}{\partial \nu_{x}} \boldsymbol{P}_{\varepsilon} f(x)
$$

on $\partial B_{\varepsilon}$. First we get

$$
\begin{aligned}
\boldsymbol{P}_{\varepsilon} f(x)= & \boldsymbol{G} f(x)+g(\boldsymbol{\varepsilon})\left(-(2 \pi)^{-1} \log \boldsymbol{\varepsilon}+\boldsymbol{\gamma}+O(\boldsymbol{\varepsilon})\right) \boldsymbol{G} f(w) \\
& +h(\boldsymbol{\varepsilon})\left((2 \pi \boldsymbol{\varepsilon})^{-1} \frac{\partial}{\partial w_{1}} G(w, y)\right)+h(\boldsymbol{\varepsilon})\left\langle\nabla_{w} S(x, w), \nabla_{w} G(w, y)\right\rangle
\end{aligned}
$$

on $x=w+(\varepsilon, 0)$. Here we notice the formulas 


$$
\begin{aligned}
& \left\langle\nabla_{w} G(x, w), \nabla_{w} G(w, y)\right\rangle_{\mid x=w+(\varepsilon, 0)} \\
& =(2 \pi \varepsilon)^{-1} \frac{\partial}{\partial w_{1}} G(w, y)+\left\langle\nabla_{w} S(x, w), \nabla_{w} G(w, y)\right\rangle \\
& \frac{\partial}{\partial x_{1}}\left\langle\nabla_{w} G(x, w), \nabla_{w} G(w, y)\right\rangle_{\mid x=w+(\varepsilon, 0)} \\
& =-(2 \pi)^{-1} \varepsilon^{-2} \frac{\partial}{\partial w_{1}} G(w, y)+\frac{\partial}{\partial x_{1}}\left\langle\nabla_{w} S(x, w), \nabla_{w} G(w, y)\right\rangle .
\end{aligned}
$$

By using relations (3.2), (3.3), (3.8), (3.9) we get the equation

$$
\begin{aligned}
(3.6)= & k(\boldsymbol{G} f(x)-\boldsymbol{G} f(w)+g(\varepsilon) O(1) \boldsymbol{G} f(w) \\
& -k^{-1} \frac{\partial}{\partial x_{1}} \boldsymbol{G} f(x)+k^{-1} \frac{\partial}{\partial w_{1}} \boldsymbol{G} f(w)+h(\varepsilon)\left\langle\nabla_{w} S(x, w), \nabla_{w} \boldsymbol{G} f(w)\right\rangle \\
& -k^{-1} h(\varepsilon) \frac{\partial}{\partial x_{1}}\left\langle\nabla_{w} S(x, w), \nabla_{w} \boldsymbol{G} f(w)\right\rangle .
\end{aligned}
$$

We know that

$$
\begin{aligned}
& g(\varepsilon)=-(2 \pi k) \varepsilon+O\left(\varepsilon^{2}|\log \varepsilon|\right) \\
& h(\varepsilon)=2 \pi \varepsilon^{2}+O\left(\varepsilon^{3}\right) .
\end{aligned}
$$

Therefore, we have (3.4).

Next we want to estimate

$$
\|L\|_{L^{2}\left(S^{1}\right)}+\|L\|_{C^{(3 / 4)+a}\left(S^{1}\right)} .
$$

We have

$$
\begin{aligned}
(3.11) & \leqq C\left(\|\boldsymbol{G} f\|_{C^{(7 / 4)+\sigma}\left(S^{1}\right)}+O\left(\varepsilon^{2}\right)\|\nabla \boldsymbol{G} f\|_{L^{\infty}(\Omega)}\right) \\
& \leqq C^{\prime}\|f\|_{L q\left(\Omega_{\varepsilon}\right)}
\end{aligned}
$$

for $q>8$. It should be remarked that $\|\boldsymbol{G} f\|_{C^{2(S 1)}}$ can not be estimated by $C\|f\|_{L q\left(\Omega_{\varepsilon}\right)}$ for any $q$. Thus, we used delicate technique of considering $H^{3 / 4}\left(S^{1}\right)$-norm. Summing up (2.2), (3.4), (3.12) we get (3.5).

\section{Proof of Theorem.}

First we consider the term $I_{l}(t)$. We have $u_{t}=v(t)+\lambda(t) \boldsymbol{P}_{t} u_{t}^{p}$, where

$$
v(t)=\lambda(t)\left(\boldsymbol{G}_{t} u_{t}^{p}-\boldsymbol{P}_{t} u_{t}^{p}\right)
$$

Therefore,

$$
I_{1}(t)=I_{1,1}(t)+I_{1,2}(t)+I_{1,3}(t),
$$

where

$$
I_{1,1}(t)=-\int_{\partial B_{t}}|\tilde{\nabla} v(t)|^{2} d \sigma_{x}
$$




$$
\begin{aligned}
& I_{1,2}(t)=-2 \lambda(t) \int_{\partial B_{t}} \tilde{\nabla} v(t) \cdot \tilde{\nabla} \boldsymbol{P}_{t} u_{t}^{p} d \sigma_{x} \\
& I_{1,3}(t)=-\lambda(t)^{2} \int_{\partial B_{t}}\left|\tilde{\nabla} \boldsymbol{P}_{t} u_{t}^{p}\right|^{2} d \sigma_{x} .
\end{aligned}
$$

We want to estimate $I_{1,1}(t)$. We know that

$$
\begin{aligned}
\tilde{V}(t) & \equiv k v(t)(x)+\frac{\partial}{\partial \nu_{x}} v(t)(x)_{\mid x \in \partial B_{t}} \\
& =\lambda(t)\left(-k \boldsymbol{P}_{t} u_{t}^{p}-\frac{\partial}{\partial \nu_{x}} \boldsymbol{P}_{t} u_{t}^{p}\right)_{\mid x \in \partial B_{t}}
\end{aligned}
$$

satisfies

$$
\begin{aligned}
& \max _{\theta}|\tilde{V}(t)(\theta)| \leqq C t\left\|u_{t}^{p}\right\|_{L q\left(\Omega_{t}\right)} \\
& \max _{x \in \partial B_{t}}|\nabla \tilde{V}(t)(x)| \leqq C t^{(1-2 \alpha) / 3}\left\|u_{t}^{p}\right\|_{L q\left(\Omega_{t}\right)}
\end{aligned}
$$

by (3.4), (3.5) for large fixed $q$.

On the other hand $u_{t}=\lambda(t)\left(\boldsymbol{G}_{t}-\boldsymbol{P}_{t}\right) u_{t}^{p}+\lambda(t) \boldsymbol{P}_{t} u_{t}^{p}$. We see that

for large $q$ and we see that

$$
\begin{aligned}
\left|\left(\boldsymbol{G}_{t}-\boldsymbol{P}_{t}\right) u_{t}^{p}\right| & \leqq C t \max _{\boldsymbol{\theta}}|L(\theta)| \\
& \leqq C^{\prime} t^{2}\left\|u_{t}^{p}\right\|_{L q\left(\Omega_{t}\right)}
\end{aligned}
$$

$$
\left|\boldsymbol{P}_{t} u_{t}^{p}\right| \leqq\left|\boldsymbol{G} \tilde{u}_{t}^{p}\right|+|g(\varepsilon)||G(x, w)|\left|\boldsymbol{G} \tilde{u}_{t}^{p}(w)\right|+|h(\varepsilon)||\nabla G(x, w)|\left|\nabla \boldsymbol{G} \tilde{u}_{t}^{p}(w)\right| .
$$

Here $\tilde{u}_{t}$ is the extension of $u_{t}$ which is zero outside $\Omega_{t}$. Since we have $\left\|u_{t}\right\|_{L p+1\left(\Omega_{t}\right)}=1$, we get $\left\|\boldsymbol{G} \tilde{u}_{t}^{p}\right\|<C^{\prime}$. Therefore, $\left|\boldsymbol{P}_{t} u_{t}^{p}\right| \leqq C^{\prime \prime}$ by observing

and

$$
\begin{aligned}
\left|\nabla \boldsymbol{G} \tilde{u}_{t}^{p}(w)\right| & \leqq C\left(\int_{\Omega_{t}}|w-y|^{-(p+1)} d y\right)^{1 /(p+1)} \\
& \leqq C^{\prime} t^{-(p-1) /(p+1)}
\end{aligned}
$$

$$
|h(t)||G(x, w)|\left|\boldsymbol{G} \tilde{u}_{t}^{p}(w)\right| \leqq C t^{1-(p-1)(p+1)-1}
$$

Summing up these fact we get

$$
\left|u_{t}\right| \leqq C+C t^{2}\left\|u_{t}^{p}\right\|_{L q\left(\Omega_{t}\right)} .
$$

By the assumption of Theorem we get

$$
\sup _{t} \sup _{x \in \Omega_{t}}\left|u_{t}(x)\right|<C^{\prime}<\infty .
$$

Then,

$$
\max _{\theta}|\tilde{V}(t)(\theta)| \leqq C^{\prime \prime} t
$$




$$
\max _{x \in \partial B_{t}}|\nabla \tilde{V}(t)(x)| \leqq C^{\prime \prime} t^{(1-2 \alpha) / 3} .
$$

Therefore, $I_{1,1}(t)=O\left(t^{1+2(1-2 \alpha) 3^{-1}}\right)$

$$
I_{1,2}(t)=O\left(t^{(1 / 2)+(1-2 \alpha) / 3}\right)\left(\int_{\partial B_{t}}\left|\tilde{\nabla} \boldsymbol{P}_{t} u_{t}^{p}\right|^{2} d \sigma_{x}\right)^{1 / 2}
$$

We know that

$$
\begin{aligned}
\int_{\partial B_{t}}\left|\tilde{\nabla} \boldsymbol{P}_{t} u_{t}^{p}\right|^{2} d \sigma_{x} \leqq & C\left(\int_{\partial B_{t}}\left|\tilde{\nabla} \boldsymbol{G} u_{t}^{p}\right|^{2} d \sigma_{x}+g(t)^{2} \int_{\partial B_{t}}\left|G(x, w) \boldsymbol{G} u_{t}^{p}(w)\right|^{2} d \boldsymbol{\sigma}_{x}\right. \\
& \left.+h(t)^{2} \int_{\partial B_{t}}\left|\left\langle\tilde{\nabla} \nabla_{w} G(x, w), \nabla_{w} \boldsymbol{G} u_{t}^{p}(w)\right\rangle\right|^{2} d \boldsymbol{\sigma}_{x}\right) .
\end{aligned}
$$

The first term in the right hand side of (4.4) is $O(t)$. The second term in the right hand side of (4.4) is $O\left(g(t)^{2}\right) t t^{-2}=O(t)$. The third term in the right hand side of (4.4) is $O\left(h(t)^{2}\right) t^{-4} t=O(t)$. Here we used the fact that $\left|\tilde{\nabla} \nabla_{w} G(x, w)\right|=$ $O\left(t^{-2}\right)$. Therefore, $I_{l}(t)=O(t)$. Thus,

$$
\int_{0}^{\varepsilon} I_{1}(t) d t=O\left(\varepsilon^{2}\right)
$$

Second we consider the term $I_{2}(t)$. By (4.1) we have $I_{2}(t)=O(t)$. Thus,

$$
\int_{0}^{\varepsilon} I_{2}(t) d t=O\left(\varepsilon^{2}\right)
$$

Similarly we have

$$
\int_{0}^{\varepsilon} I_{3}(t) d t=O\left(\varepsilon^{2}\right)
$$

We would like to consider the integral of $I_{4}(t)$ from 0 to $\varepsilon$ which is a main term of our analysis. We have $u_{t}=\lambda(t) \boldsymbol{G}_{t} u_{t}^{p}$ we get $I_{4}(t)=O(1)$. Thus, we have

$$
\int_{0}^{\varepsilon} I_{4}(t) d t=O(\varepsilon) \text {. }
$$

Summing up these estimates we have

$$
\lambda(\varepsilon)-\lambda=O(\varepsilon) .
$$

We need more delicate analysis to get Theorem. We have

$$
\left(\int_{\partial B_{t}} u_{t}^{2} d \sigma_{x}\right)=\tilde{I}_{5}(t)+\tilde{I}_{6}(t)+\tilde{I}_{7}(t)
$$

where

$$
\begin{aligned}
& \tilde{I}_{5}(t)=\lambda(t)^{2} \int_{\partial B_{t}}\left(\boldsymbol{P}_{t} u_{t}^{p}\right)^{2} d \boldsymbol{\sigma}_{x} \\
& \tilde{I}_{6}(t)=2 \lambda(t)^{2} \int_{\partial B_{t}}\left(\boldsymbol{P}_{t} u_{t}^{p}\right)\left(\boldsymbol{G}_{t}-\boldsymbol{P}_{t}\right) u_{t}^{p} d \boldsymbol{\sigma}_{x}
\end{aligned}
$$




$$
\tilde{I}_{7}(t)=\lambda(t)^{2} \int_{\partial B_{t}}\left(\left(\boldsymbol{G}_{t}-\boldsymbol{P}_{t}\right) u_{t}^{p}\right)^{2} d \boldsymbol{\sigma}_{x} .
$$

Since we have (3.4), Lemma 2.1 we get

$$
\left|\left(\boldsymbol{G}_{t}-\boldsymbol{P}_{t}\right) u_{t}^{p}\right| \leqq C^{\prime \prime} t^{2}\left\|u_{t}^{p}\right\|_{L q\left(\Omega_{t}\right)} \leqq C t^{2} .
$$

Therefore,

$$
\tilde{I}_{6}(t) \leqq C\left(\int_{\partial B_{t}}\left(\boldsymbol{P}_{t} u_{t}^{p}\right)^{2} d \boldsymbol{\sigma}_{x}\right)^{1 / 2} t^{5 / 2} \leqq \tilde{C} \tilde{I}_{5}(t)^{1 / 2} t^{5 / 2}
$$

by the Schwartz inequality. We have

$$
\tilde{I}_{7}(t)=O\left(t^{5}\right) .
$$

We want to calculate $\tilde{I}_{5}(t)$.

$$
\begin{aligned}
\tilde{I}_{5}(t)= & \lambda(t)^{2} \int_{\partial B_{t}}\left(\boldsymbol{G} \tilde{u}_{t}^{p}\right)(x)^{2} d \boldsymbol{\sigma}_{x} \\
& +2 \lambda(t)^{2} \int_{\partial B_{t}} \boldsymbol{G} \tilde{u}_{t}^{p}(x) g(t) G(x, w) \boldsymbol{G} \tilde{u}_{t}^{p}(w) d \boldsymbol{\sigma}_{x} \\
& +2 \lambda(t)^{2} \int_{\partial B_{t}} \boldsymbol{G} \tilde{u}_{t}^{p}(x) h(t)\left\langle\nabla_{w} G(x, w), \nabla_{w} \boldsymbol{G} \tilde{u}_{t}^{p}(w)\right\rangle d \sigma_{x} \\
& +\lambda(t)^{2} g(t)^{2} \int_{\partial B_{t}} G(x, w)^{2}\left(\boldsymbol{G} \tilde{u}_{t}^{p}\right)(w)^{2} d \sigma_{x} \\
& +2 \lambda(t) g(t) h(t) \int_{\partial B_{t}} G(x, w) \boldsymbol{G} \tilde{u}_{t}^{p}(w)\left\langle\nabla_{w} G(x, w), \nabla_{w} \boldsymbol{G} u_{t}^{p}(w)\right\rangle d \sigma_{x} \\
& +\lambda(t)^{2} h(t)^{2} \int_{\partial B_{t}}\left\langle\nabla_{w} G(x, w), \nabla_{w} \boldsymbol{G} u_{t}^{p}(w)\right\rangle^{2} d \sigma_{x} \\
= & \tilde{I}_{8}(t)+\tilde{I}_{9}(t)+\cdots+\tilde{I}_{13}(t) .
\end{aligned}
$$

We have $\tilde{I}_{9}(t)=O\left(t^{2}|\log t|\right), \quad \tilde{I}_{10}(t)=O\left(t^{2}\right), \quad \tilde{I}_{11}(t)=O\left(t^{3}(\log t)^{2}\right), \quad \tilde{I}_{12}(t)=O\left(t^{3}|\log t|\right)$, $\tilde{I}_{13}(t)=O\left(t^{3}\right)$. Since we have (4.9) we get

$$
\tilde{I}_{8}(t)=\lambda^{2} \int_{\partial B_{t}}\left(\boldsymbol{G} \tilde{u}_{t}^{p}\right)(x)^{2} d \sigma_{x}+O\left(t^{2}\right)
$$

Thus,

$$
\tilde{I}_{5}(t)=\lambda^{2} \int_{\partial B_{t}}\left(\boldsymbol{G} \tilde{u}_{t}^{p}\right)(x)^{2} d \sigma_{x}+O\left(t^{2}\right)=O(t)
$$

Thus, $\tilde{I}_{6}(t)=O\left(t^{3}\right)$. Therefore,

$$
I_{4}(t)=k \lambda^{2} t^{-1} \int_{\partial B_{t}} G \tilde{u}_{t}^{p}(x)^{2} d \sigma_{x}+O(t) .
$$

Summing up these facts we get 


$$
\lambda(\varepsilon)-\lambda=k \lambda^{2} \int_{0}^{\varepsilon}\left(t^{-1} \int_{\partial B_{t}} G \tilde{u}_{t}^{p}(x)^{2} d \sigma_{x}\right) d t+O\left(\varepsilon^{2}\right) .
$$

By the assumption of Theorem we have $\boldsymbol{G} \tilde{u}_{t}^{p}(x)-\boldsymbol{G} \boldsymbol{u}^{p}(x)=o(1)$ uniformly for $x$. Therefore we get Theorem.

\section{Comments.}

We know that the condition

$$
\sup _{\varepsilon} \varepsilon^{2}\left\|\boldsymbol{u}_{\varepsilon}^{p}\right\|_{L q\left(\Omega_{\varepsilon}\right)}<C^{\prime}<+\infty
$$

in Theorem can be replaced by

$$
\sup _{\varepsilon} \sup _{x \in \Omega_{\varepsilon}}\left|u_{\varepsilon}(x)\right|<C<+\infty .
$$

The author conjectures that (5.1) follows from other conditions in Theorem.

Our proof of Theorem of this paper is quite different from the proof of Theorem 1 (with $\sigma=0$ ) in Ozawa [11]. Our proof of this paper used Hadamard's variational formula for non-linear eigenvalue in [7].

The authors want to get the asymptotic estimate of eigenvalues of $q$ Laplacian under singular variation of domains. Here this problem is related to minimizing problem of

$$
\inf _{u \in X} \int_{\Omega}|\nabla u|^{q} d x
$$

where $X=\left\{\|u\|_{L^{p+1}(\Omega)}=1, u \in W^{1, q}(\Omega), u=0\right.$ on $\left.\partial \Omega\right\}$. However the Euler equation is complicated compared with the case $q=2$. Can one get any result?

\section{REFERENCES}

[1] R. Adams, Sobolev spaces, Academic Press, New York, 1975.

[2] G. BEsson, Comportment asymptotique des valeurs propres du Laplacien dans un domaine avec un trou, Bull. Soc. Math. France., 113 (1985), 211-239.

[3] H. Berestychi, P.L. Lions, L. A. Peletiet, An ODE approach to the existence of positive solution for semilinear problems in $R^{N}$, Indiana Univ. Math. J. 30 (1981), 141-157.

[4] E.N. DANCER, The effect of domain shape on the number of positive solutions of certain nonlinear equations, J. Diff. Equations., 74 (1988), 120-156.

[5] S.S. LiN, Semilinear elliptic equations on singularly perturbed domains, Commun. in Partial Diff. Equations., 16 (1991), 617-645.

[6] W.M. Ni, J. SERrin, Nonexistence theorem for singular solutions of quasilinear partial differential equations, Comm. Pure Appl. Math., 34 (1986), 379-399.

[7] T. Osawa, The Hadamard variational formula for the grand state value of $-\Delta u=\lambda|u|^{p-1} u$, Kodai Math, J. 15 (1992), 258-278.

[8] S. OzAwA, Electrostatic capacity and eigenvalues of the Laplacian, J. Fac. Sci. 
Univ. Tokyo Sec IA., 30 (1983), 53-62.

[9] S. OzAwa, Spectra of domains with small spherical Neumann boundary, Ibid., 30 (1983), 259-277.

[10] S. OzAwA, Asymptotic property of an eigenfunction of the Laplacian under singular variation of domains, -the Neumann condition-, Osaka J. Math., 22 (1985), 639-655.

[11] S. OzAwA, Singular variation of domain and spectra of the Laplacian with small Robin conditional boundary I. to appear in Osaka I. Math.

[12] S. OzAwa, Fluctuation of spectra in random media. II Ibid., 27 (1990), 17-66.

[13] S. OzAwa, Singular variation of nonlinear eigenvalue, Proc. Japan Acad. 68A (1992), 154-156.

[14] P.H. Rabinowitz, Variational methods for nonlinear elliptic eigenvalue problems, Indian Univ. Math. J. 23 (1974), 729-754.

[15] X.J. WANG, Neumann problem of semilinear elliptic equations involving critical Sobolev exponents. J. of Diff. Equations., 93 (1991), 283-310.

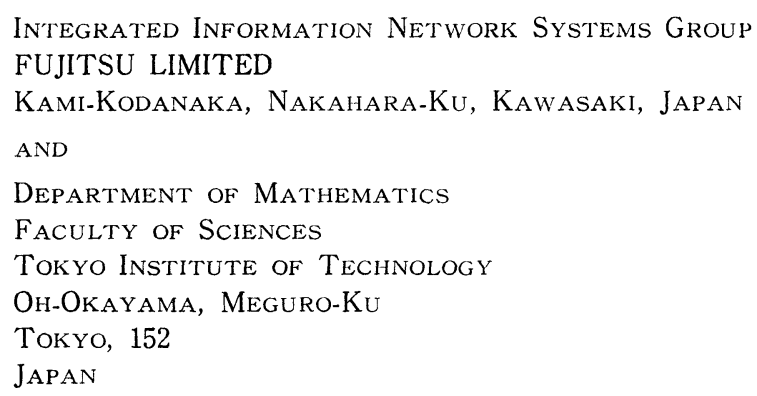

Addendum: Right hand side of formula (3.4) should be corrected as $C \varepsilon^{h}\|f\|_{L}{ }^{p}\left(\Omega_{\varepsilon}\right)$ for $h<1$ and large $q$. And it suffices to get our Theorem, if an assumption of Theorem, which is

$$
\sup _{\varepsilon} \varepsilon^{2}\left\|u_{\varepsilon}^{p}\right\|_{L}^{p}\left(\Omega_{\varepsilon}\right)<C<+\infty
$$

for large $q$ is replaced by

$$
\sup _{\varepsilon} \varepsilon^{1+h}\left\|u_{\varepsilon}^{p}\right\|_{L^{p}\left(\Omega_{\varepsilon}\right)}<C_{q, h}^{\prime}<+\infty
$$

for any $h<1$ and large $q$. 